\title{
Clinical evolution, management, and resolution of type II necrotizing fasciitis
}

\author{
Jina Makadia $\cdot$ Nitin Bhanot . \\ Sheri A. Mancini $\cdot$ Zaw Min
}

Received: 12 September 2014/ Accepted: 26 September 2014/Published online: 8 October 2014

(C) SIMI 2014

\begin{abstract}
A 60-year-old woman with a history of pre-diabetes and hyperlipidemia, presented to a local hospital with severe left flank pain and skin discoloration on the same area for 2 days. She had been taking care of her grandson who was diagnosed with streptococcal sore throat. On examination, the patient was hypotensive and tachycardic. There was a large grayish blue area over the left flank, extending to the left lower back (Fig. 1a, b). This area was very tender to palpation. There were no bullae, crepitus, or external drainage. A CT scan of the abdomen revealed a diffuse inflammatory process within the skin and soft tissue without any gas in the adjacent area. A diagnosis of necrotizing fasciitis was pursued. Broad-spectrum intravenous antibiotics (vancomycin, piperacillin-tazobactam, and clindamycin), and vasopressors were initiated, and she was transferred to our institution for further management. Upon arrival, she was immediately taken to the operating room for surgery. The operative findings showed there were
\end{abstract}

J. Makadia and Z. Min equally contributed to this manuscript.

J. Makadia $\cdot$ N. Bhanot $\cdot$ Z. Min $(\bowtie)$

Department of Medicine, Division of Infectious Diseases, Allegheny General Hospital, Allegheny Health Network,

420 East North Avenue, East Wing, Suite 407, Pittsburgh, PA 15212, USA

e-mail: zmin@wpahs.org

J. Makadia

e-mail: jmakadia@wpahs.org

N. Bhanot

e-mail: nbhanot@wphas.org

\section{S. A. Mancini}

Department of Surgery, Allegheny General Hospital,

Allegheny Health Network, 420 East North Avenue, Suite 304,

Pittsburgh, PA 15212, USA

e-mail: smancini@wpahs.org extensive soft tissue necrosis and edematous fascial layers. A full-thickness debridement of skin and subcutaneous tissue of the left flank $(24 \mathrm{~cm} \times 21 \mathrm{~cm} \times 1.5 \mathrm{~cm})$ was performed (Fig. 1c). The tissue culture was positive for group A $\beta$-hemolytic Streptococcus (Streptococcus pyogenes). The pathogen was sensitive to penicillin (minimal inhibitory concentration, MIC $0.032 \mathrm{mg} / \mathrm{L}$ ), ceftriaxone (MIC $0.064 \mathrm{mg} / \mathrm{L}$ ), vancomycin (MIC $1.0 \mathrm{mg} / \mathrm{L}$ ), and clindamycin (MIC not reported). Blood cultures were negative throughout the admission. Antibiotics were tailored to intravenous penicillin and clindamycin.

Despite appropriate antimicrobial therapy, on day 3 of hospitalization, there were new areas of purplish red discoloration changes noted around the edge of the wound (Fig. 1d). She underwent additional debridement of skin and subcutaneous tissues (Fig. 1e). A day later (day 4 of hospitalization), it was again noted that the infection was extending upward to the left axilla at which point further debridement was undertaken (Fig. 1f). On day 6 of admission, extension of bluish gray skin changes occurred medially towards the suprapubic region (Fig. $1 \mathrm{~g}$ ), requiring an additional large area $(20 \mathrm{~cm} \times 11 \mathrm{~cm} \times 2 \mathrm{~cm})$ of surgical debridement (Fig. 1h). The negative pressure wound therapy devices were applied to the wounds, and the wound dressings were regularly changed under anesthesia. Vasopressors were successfully weaned off, and she continued to improve. On day 20 of hospitalization, the patient was eventually discharged home with negative pressure wound therapy. Approximately 2 months after the initial hospitalization, the abdominal and axillary wounds were found to be sufficiently granulated (Fig. 1i). She underwent definitive wound closure with split-thickness skin grafts harvested from the left thigh. One month post-skin graft follow-up (day 84 from the first hospitalization), skin grafts were taken very well without further complications (Fig. 1j). 

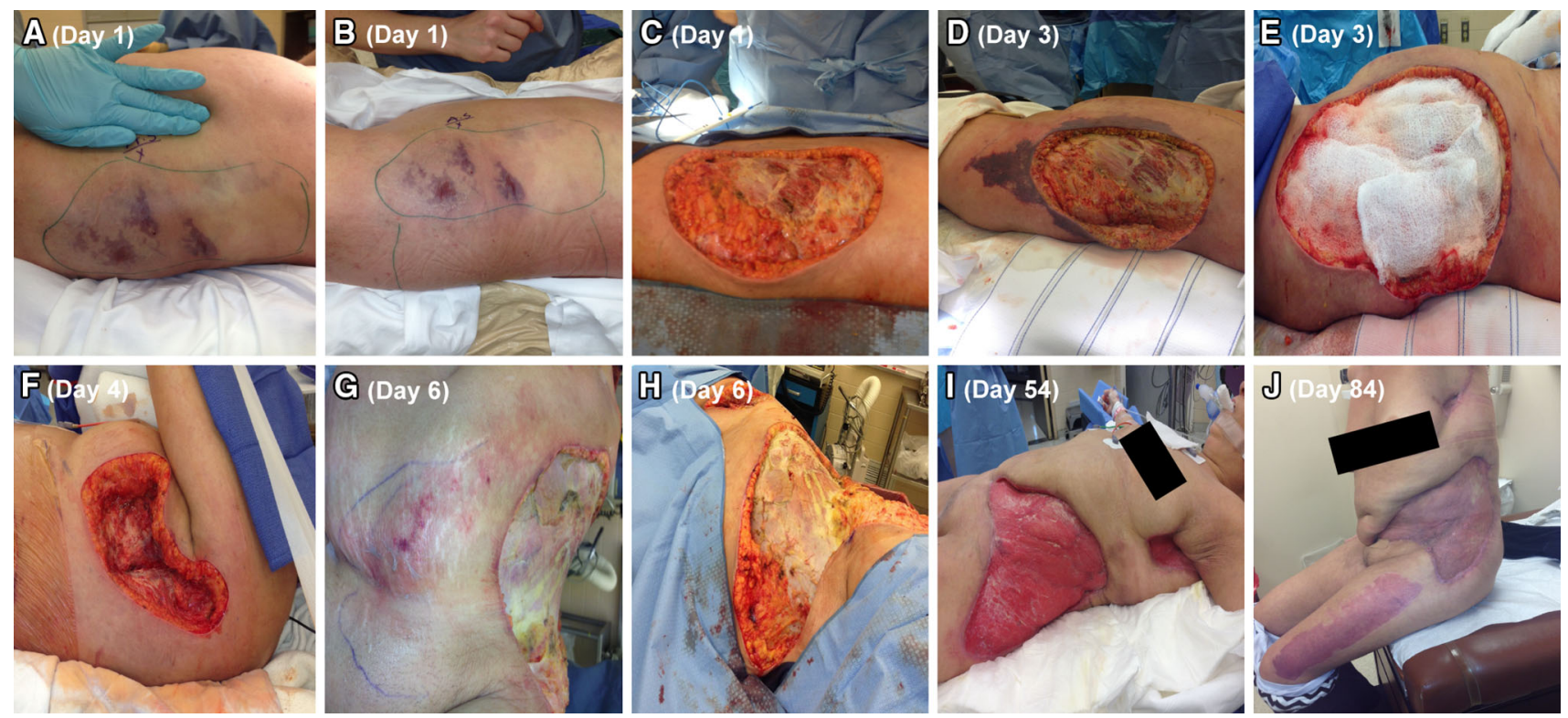

Fig. 1 The photos illustrated remarkable progression of streptococcal necrotizing fasciitis of left abdominal wall and left axilla (a through $\mathbf{h})$. i, j represented pre- and post-skin grafts. Days were calculated from the first day of hospitalization

Necrotizing fasciitis is a grievous infection of the deeper skin, subcutaneous tissues and fascia $[1,2]$. It is characterized by extensive and rapidly spreading necrosis and gangrene of the subcutaneous fat and fascia. There are two distinct types of necrotizing fasciitis depending on the causal pathogens [1]. Type I necrotizing fasciitis is caused by polymicrobial flora, including at least one anaerobic bacterium (Bacteroides, Clostridium, or Peptostreptococcus), together with anaerobic streptococci (other than group A), and Enterobacteriaceae. On the other hand, type II necrotizing fasciitis is secondary to monomicrobial infection, and the majority of cases are due to infection of group A $\beta$ hemolytic Streptococcus (GAS; Streptococcus pyogenes) and, therefore, it is known as hemolytic streptococcal gangrene. Other important pathogens known to cause monomicrobial necrotizing fasciitis include methicillin-resistant Staphylococcus aureus (MRSA), Vibrio vulnificus, and Aeromonas hydrophilia [1, 2]. Recently, necrotizing soft tissue infection secondary to V. vulnificus has been proposed to be a distinct category (type III necrotizing fasciitis) [1].

In 2012, the United States Centers of Disease Control and Prevention (CDC) reported a total of 1,117 patients (3.4 cases per 100,000 population) with invasive GAS infection. Among them, 75 cases $(6.7 \%)$ were identified as necrotizing fasciitis caused by GAS [3]. Common predisposing factors are diabetes mellitus, alcoholism, peripheral vascular disease, obesity, recent surgery, traumatic wounds, cirrhosis, corticosteroid therapy, and immunosuppression [1, 4]. Except for surgical and traumatic wounds, the portal of pathogen entry in other cases is usually from at a site of trivial trauma (minor scratches, or insect bites), and most of the patients do not recall this inapparent injury [1, 2, 4]. Most infections are localized to a lower extremity or the trunk [1].

The initial clinical presentation appears just as an area of mild erythema. Then, it undergoes a rapid evolution over the next $24-48 \mathrm{~h}$, at which time the affected skin color tends to become purplish red, dusky, ecchymosis, or mottled $[2,4]$. The affected site is exquisitely tender, and pain disproportionate to physical examination is a classical finding in this stage [1, 2, 4]. Another distinct clinical feature is woody hard induration on palpation because the uninvolved underlying tissues are firm and the deeper infected fascial layers cannot be elicited by palpation [2]. If diagnosis is missed at this stage, affected cutaneous area quickly progresses to breakdown with bullae containing yellow or hemorrhagic fluid, and obvious cutaneous gangrene ensues. By this time, the involved area is no longer tender, but becomes numb from infarction of superficial cutaneous nerves secondary to deprivation of the blood supply $[1,2]$.

Laboratory findings are usually non-specific, and the major challenge of managing necrotizing fasciitis remains establishing a rapid clinical diagnosis. It is prudent to think of the diagnosis of necrotizing fasciitis in a high risk patient with cutaneous infection presenting as erythema, pain out of proportion to examination, or tenderness extending beyond the apparent areas of skin involvement, and to rapidly and aggressively institute therapy. Rather than delaying to obtain radiographic imaging, prompt surgical exploration is highly recommended in cases of possible necrotizing fasciitis for both diagnostic and 
therapeutic purposes $[1,2,4]$. Typical surgical findings include characteristic "dishwater" murky fluid, necrotic tissues, absence of bleeding, vascular thrombosis, and a positive "finger test" (lack of resistance to finger dissection through the fascial layers) $[1,2]$. Extensive surgical debridement is usually required because necrosis and inflammation track along the fascial layers extending beyond the area of apparent clinical finding [1].

Initial administration of broad-spectrum antibiotics to cover MRSA, Streptococcus, Gram-negative pathogens, and anaerobes is required in patients with suspected necrotizing soft tissue infections $[1,2]$. The antimicrobial therapy can then be streamlined to high dose intravenous penicillin and clindamycin once GAS is confirmed as the causal pathogen. The addition of clindamycin is thought to neutralize the effect of streptococcal exotoxins and systemic cytokine inflammatory response, thus blocking the progression to streptococcal toxic shock syndrome $[1,2,4]$. Antimicrobial therapy should be continued until adequate surgical procedures have been performed, obvious clinical improvement is observed, and there is an absence of fever for $48-72 \mathrm{~h}$ [1]. Other adjuvant therapies may include administration of intravenous immunoglobulins (IVIG) and hyperbaric oxygen therapy though their use is not universal $[2,4]$. Overall, aggressive early surgical debridement is central to the management of necrotizing fasciitis, whereas antibiotics and other therapies are regarded as ancillary therapeutic modalities. Even with optimal management, the mortality from streptococcal necrotizing fasciitis is high (30-70\%) [2]. Antibiotic therapy alone, without surgical debridement, carries a mortality rate approaching $100 \%$ $[2,4]$.

In conclusion, GAS necrotizing soft tissue infection is a highly fatal infectious disease. The patient may only present with skin erythema, but an important clue to necrotizing fasciitis is the amount of pain out of proportion to the apparent degree of illness in patients with diabetes, cirrhosis, or immune suppression. A high index of clinical suspicion is critical in making an early diagnosis of necrotizing fasciitis before the late manifestations of the infection become clear since outcome is time constrained. Our case highlights this importance of recognition of rapid evolution of subtle cutaneous changes in GAS necrotizing fasciitis. Timely aggressive surgical intervention in our case was crucial for both diagnosis and improving the patients' survival. Targeted antimicrobial therapy and other therapeutic agents are regarded as important supportive adjuvants.

Acknowledgments All authors extend our sincere thanks to the patient who provided us explicit verbal and written consents to use clinical case and her photos in this publication for medical education, research, and teaching.

Conflict of interest None.

\section{References}

1. Pasternack MS, Swartz MN (2014) Cellulitis, necrotizing fasciitis, and subcutaneous tissue infections. In: Bennett J, Dolin R, Blaser M (eds) Mandell, Douglas, and Bennett's principles and practice of infectious diseases, 8th edn. Saunders, Philadelphia, pp 1211-1215

2. Stevens DL, Bisno AL, Chambers HF, Dellinger EP, Goldstein EJ, Gorbach SL, Hirschmann JV, Kaplan SL, Montoya JG, Wade JC (2014) Practice guidelines for the diagnosis and management of skin and soft tissue infections: 2014 update by the infectious diseases society of America. Clin Infect Dis 59(2):e10-e52

3. The US Centers for Disease Control and Prevention (2013) Active Bacterial Core Surveillance Report, Emerging Infections Program Network, Group A Streptococcus, 2012. http://www.cdc.gov/abcs/ reports-findings/survreports/gas12.pdf. Accessed 08 Sep 2014

4. Anaya DA, Dellinger EP (2007) Necrotizing soft-tissue infection: diagnosis and management. Clin Infect Dis 44(5):705-710 